\title{
Prescription of antibiotics after tooth extraction in adults: a nationwide study in Korea
}

\author{
Yoon-Young Choi ${ }^{1,2}$ \\ ${ }^{1}$ Graduate School of Clinical Dentistry, Korea University, Seoul, ${ }^{2}$ Private Practice, Anyang, Korea
}

\begin{abstract}
J Korean Assoc Oral Maxillofac Surg 2020;46:49-57)
Objectives: This study aimed to understand the nationwide patterns of antibiotic prescription after tooth extraction in adult patients. Materials and Methods: This study analyzed dental records from the National Health Insurance Service-National Sample Cohort (NHIS-NSC) database on 503,725 tooth extractions performed in adults ( $\geq 19$ years) during 2011-2015. Patient sex, age, household income, systemic disease (diabetes mellitus and hypertension), type of dental institution, region of dental institution, year of prescription, and type of tooth extraction procedure were considered. The antibiotic prescription rate and broad-spectrum antibiotic prescription frequency were analyzed using chi-squared tests. Factors affecting the prescription of broad-spectrum antibiotics were evaluated using multivariate logistic regression analysis.

Results: The rate of antibiotic prescription after tooth extraction was $81.85 \%$. Penicillin was most commonly prescribed (45.25\%), followed by penicillin with beta-lactamase inhibitors (18.76\%), metronidazole (12.29\%), and second- to fourth-generation cephalosporins (11.52\%). The proportion of broad-spectrum antibiotics used among all prescribed antibiotics was $45.88 \%$.

Conclusion: The findings of this study demonstrate that the rate of antibiotic prescription after tooth extraction is higher in Korea than in other countries. Furthermore, broad-spectrum antibiotics are used more frequently, which may indicate unnecessary drug prescription, an important contributor to antibiotic resistance.
\end{abstract}

Key words: Anti-bacterial agents, Antibiotics, Korea, Prescriptions, Tooth extraction

[paper submitted 2019. 9. 23 / accepted 2019. 10. 28]

\section{Introduction}

Antibiotics are commonly prescribed in dental clinics along with nonsteroidal anti-inflammatory drugs ${ }^{1}$. In dental clinics, antibiotics are often prescribed to assist with surgical procedures, treat inflammatory conditions such as acute ulcerative gingivitis, and prevent systemic infections such as bacterial endocarditis ${ }^{2,3}$. However, misuse of antibiotics has highlighted the issue of antibiotic resistance, to the extent that a warning was announced by the World Health Organization ${ }^{4}$. A recent study reported that infection with antibiotic-resistant bacteria may kill 10 million people every year until $2050^{5}$. In

\section{Yoon-Young Choi \\ Private Practice, 180 Simin-daero, Dongan-gu, Anyang 14073, Korea TEL: +82-31-8086-9898 FAX: +82-31-8086-9899 \\ E-mail:dencyy@naver.com \\ ORCID: https://orcid.org/0000-0002-7973-719X}

(2) This is an open-access article distributed under the terms of the Creative Commons Attribution Non-Commercial License (http://creativecommons.org/ licenses/by-nc/4.0/), which permits unrestricted non-commercial use, distribution, and reproduction in any medium, provided the original work is properly cited. Copyright (C) 2020 The Korean Association of Oral and Maxillofacial Surgeons. All rights reserved. addition, another study that assessed Staphylococcus aureus in saliva samples collected from 122 participants showed that $88.6 \%$ of the strain exhibited resistance to two or more antibiotics ${ }^{6}$.

Among all antibiotic prescriptions, the proportion of prescriptions from dental clinics was reported to be approximately $10 \%{ }^{3,7-9}$. Several previous studies have reported inappropriate prescription of antibiotics in dental clinics ${ }^{10,11}$, and the high rate of broad-spectrum antibiotic prescription in dental clinics has been identified as a cause of antibiotic resistance ${ }^{12}$. In order to prevent antibiotic-resistance development caused by the misuse of antibiotics, broad-spectrum antibiotic use should be limited to cases of severe infection ${ }^{13}$.

In dental clinics, antibiotics are frequently prescribed to prevent local and systemic infections that may occur after invasive surgical treatment. Among various invasive procedures, the rate of antibiotic prescription is particularly high after tooth extractions ${ }^{14,15}$. However, indiscriminate prescription of antibiotics to all patients after tooth extraction has been highlighted as antibiotic misuse, particularly in patients who have a very low risk of infection (e.g., simple tooth ex- 
traction in a healthy patient without systemic disease) ${ }^{16}$.

Antibiotic prescription patterns in dental clinics have been described for various countries. A previous study that analyzed antibiotic prescription in Korean dental clinics reported that antibiotics prescribed after tooth extractions account for the greatest proportion of antibiotic use following dental procedures $^{17}$. A nationwide study that analyzed antibiotic prescriptions from dentists in Germany over a 4-year period reported that, although the most frequently prescribed antibiotic was amoxicillin, the rate of prescribing clindamycin (a broad-spectrum antibiotic) increased over the study period, and the rate of prescribing clindamycin in the most recent year was markedly higher than the rate prescribed in medical clinics $^{12}$. A retrospective cross-sectional study in the United States analyzed the medical records of antibiotic prescription in 2015. Similar to the German study, amoxicillin was the most frequently prescribed antibiotic, but broad-spectrum antibiotics such as amoxicillin clavulanate or clindamycin were frequently used and the prescription duration was longer than that in medical clinics?.

Studies assessing the status of antibiotic prescription in dental clinics are essential to resolve the issue of antibiotic misuse. In Korea, enrollment in the National Health Insurance Service (NHIS) is enforced by law, and $\geq 98 \%$ of the citizens are enrolled in this service. The National Health Insurance Service-National Sample Cohort (NHIS-NSC) is a dataset comprised of data representing $2 \%$ of the entire Korean population and involves stratified sampling based on sex, age, health insurance cost, and region. In addition, this vast database includes information on not only the use of medical services, but also on sociodemographic factors and family relationships of the population. Furthermore, detailed information regarding medications prescribed at medical and dental clinics is included, making this database an optimal source for the analysis of antibiotic prescriptions in dental clinics. To the best of our knowledge, no previous study has assessed antibiotic prescription after tooth extraction using the Korean NHIS-NSC database. Thus, this study aimed to utilize the NHIS-NSC database to understand the nationwide patterns of antibiotic prescription after tooth extraction and to identify factors affecting the prescription of broad-spectrum antibiotics in Korea.

\section{Materials and Methods}

\section{Study participants}

Among the NHIS-NSC participants, adults $\geq 19$ years of age who underwent tooth extraction in dental institutions in Korea between 2011 and 2015 were included in this study. Of the 528,483 cases of tooth extraction that met the inclusion criteria, cases with missing values for variables were excluded. A total of 503,725 cases were included in this study. (Table 1)

\section{Variables}

Explanatory variables in this study included patient sex, age, household income, systemic disease (diabetes mellitus and hypertension), type of dental institution, region of dental institution, year of prescription, and type of tooth extraction procedure. The patients were divided into the following age groups: 19-30, 31-40, 41-50, 51-60, 61-70, and $\geq 71$ years, and the household income was classified into quintiles based on the health insurance premium. Systemic disease in patients was assessed using medical records containing the codes for diabetes (E10-E14) and hypertension (I10-I15) based on the International Classification of Disease, 10th revision (ICD10) between 2011 and $2015^{18}$. The types of dental institutions were divided into dental hospitals or dental clinics, and the region of the dental institution was divided into rural or urban areas. The type of tooth extraction was classified according to NHIS treatment codes: U4412 (incisor extraction), U4413 (molar extraction), U4414 (complicated extraction: although not impacted, simple extraction is not possible due to dental root hypertrophy, root dilacerations, or osseointegration), U4415 (simply impacted tooth), U4416 (complexly impacted tooth: cases involving tooth splitting), and U4417 (completely impacted tooth: cases involving simultaneous tooth split-

Table 1. Summary of patient characteristics

\begin{tabular}{lc}
\hline \multicolumn{1}{c}{ Characteristic } & Value \\
\hline Total number of tooth extraction cases & 503,725 \\
in this study & \\
Cases with prescription & 443,473 \\
Cases with antibiotic prescription & 412,308 \\
Cases with more than two antibiotics & 2,588 \\
prescribed & 252,549 \\
Total number of patients & $50.42 \pm 17.43$ \\
Mean patient age (yr) & \\
\hline Values are presented as number only or mean \pm standard deviation. \\
Yoon-Young Choi: Prescription of antibiotics after tooth extraction in adults: a nationwide \\
study in Korea. J Korean Assoc Oral Maxillofac Surg 2020
\end{tabular}


ting and ostectomy of impacted tooth with $\geq 2 / 3$ of crown impacted into alveolar bone). If more than two types of tooth extractions were performed in a single day, the more invasive technique was included.

\section{Types of antibiotics}

Types (groups) of antibiotics were classified as follows based on the Anatomical Therapeutic Chemical (ATC) classification system ${ }^{19}$ and with reference to a previous study ${ }^{20}$ : penicillins, first-generation cephalosporins, tetracyclines, penicillins with beta-lactamase inhibitors, metronidazole, second- to fourth-generation cephalosporins, macrolides, quinolones, lincomycin-derivatives, and others. Among these, penicillins with beta-lactamase inhibitors, metronidazole, second- to fourth-generation cephalosporins, macrolides, quinolones, and lincomycin-derivatives were defined as broadspectrum antibiotics ${ }^{13,21,22}$. If two or more antibiotics were prescribed at a single visit, the case was classified as broadspectrum antibiotic prescription.

\section{Data analysis}

Chi-squared tests were performed to compare the types of antibiotics prescribed for different types of tooth extraction procedures and to compare the rates of antibiotic prescription and broad-spectrum antibiotic prescription. Furthermore, to identify the factors affecting the prescription of broad-spec- trum antibiotics, a multivariate logistic regression analysis was performed. All statistical analyses were performed using SAS Statistics (ver. 9.2; SAS Institute, Cary, NC, USA), and $P$-values of $<0.05$ were considered significant.

\section{Ethics approval}

This study was performed after receiving ethics approval from the Public Institutional Review Board Committee (P01201901-21-004), and all experimental procedures were conducted in compliance with the Declaration of Helsinki. Data from the NHIS-NSC are reconstituted data that do not include personal identification, and thus the need for obtaining informed consent from subjects was waived by the institutional review board committee.

\section{Results}

This study included 503,725 tooth extraction cases, of which 443,473 were given prescriptions. Among them, 412,308 cases had prescriptions that included antibiotics in 252,549 unique patients.(Table 1)

\section{Types of antibiotics prescribed for different tooth extraction procedures}

Overall, the most common type of antibiotic prescribed after tooth extraction was penicillin (45.25\%) (Table 2$)$, fol-

Table 2. Types of antibiotics in different tooth extraction procedures

\begin{tabular}{|c|c|c|c|c|c|c|c|}
\hline Division & $\begin{array}{l}\text { Incisor } \\
\text { extraction }\end{array}$ & $\begin{array}{l}\text { Molar } \\
\text { extraction }\end{array}$ & $\begin{array}{l}\text { Complicated } \\
\text { extraction }^{1}\end{array}$ & $\begin{array}{c}\text { Simply } \\
\text { impacted tooth }\end{array}$ & $\begin{array}{c}\text { Complexly } \\
\text { impacted tooth }\end{array}$ & $\begin{array}{c}\text { Completely } \\
\text { impacted tooth }\end{array}$ & Total \\
\hline Penicillins & $22,471(46.47)$ & $127,485(47.21)$ & $18,813(43.59)$ & $6,108(42.24)$ & $6,263(37.92)$ & $6,673(29.55)$ & $187,813(45.25)$ \\
\hline $\begin{array}{l}\text { Penicillins with beta- } \\
\text { lactamase inhibitors }\end{array}$ & $7,655(15.83)$ & $46,910(17.37)$ & $8,848(20.50)$ & $3,338(23.08)$ & $4,504(27.27)$ & $6,630(29.36)$ & 77,885 (18.76) \\
\hline Metronidazole & $6,775(14.01)$ & $34,590(12.81)$ & $5,394(12.50)$ & $1,422(9.83)$ & $1,259(7.62)$ & $1,585(7.02)$ & $51,025(12.29)$ \\
\hline $\begin{array}{l}\text { Second- to fourth- } \\
\text { generation } \\
\text { cephalosporins }\end{array}$ & 4,997 (10.33) & $28,775(10.66)$ & $5,372(12.45)$ & $1,847(12.77)$ & $2,521(15.26)$ & $4,320(19.13)$ & $47,832(11.52)$ \\
\hline $\begin{array}{l}\text { First-generation } \\
\text { cephalosporins }\end{array}$ & $4,358(9.01)$ & $22,019(8.15)$ & $3,475(8.05)$ & $1,381(9.55)$ & $1,613(9.77)$ & $2,898(12.83)$ & $35,744(8.61)$ \\
\hline Macrolides & $886(1.83)$ & 4,561 (1.69) & $615(1.43)$ & $164(1.13)$ & $162(0.98)$ & $173(0.77)$ & $6,561(1.58)$ \\
\hline Quinolones & $798(1.65)$ & $3,785(1.40)$ & $447(1.04)$ & $154(1.06)$ & $157(0.95)$ & $219(0.97)$ & $5,560(1.34)$ \\
\hline $\begin{array}{l}\text { Lincomycin } \\
\text { derivatives }\end{array}$ & $121(0.25)$ & $566(0.21)$ & $82(0.19)$ & $26(0.18)$ & $25(0.15)$ & $55(0.24)$ & $875(0.21)$ \\
\hline Tetracyclines & $280(0.58)$ & $1,283(0.48)$ & $109(0.25)$ & $21(0.15)$ & $12(0.07)$ & $21(0.09)$ & $1,726(0.42)$ \\
\hline Others & $20(0.04)$ & $43(0.02)$ & $2(0.00)$ & $0(0.00)$ & $1(0.01)$ & $6(0.03)$ & $72(0.02)$ \\
\hline
\end{tabular}

${ }^{1}$ Cases where simple extraction was not possible due to root hypertrophy, root dilacerations, or osseointegration.

${ }^{2}$ Cases involving tooth splitting.

${ }^{3}$ Simultaneous tooth splitting and ostectomy of impacted tooth with $\geq 2 / 3$ of crown impacted into alveolar bone.

$P<0.001, \chi^{2}=8263.89$ ( $P$-value calculated using chi-squared test).

Values are presented as number $(\%)$.

Yoon-Young Choi: Prescription of antibiotics after tooth extraction in adults: a nationwide study in Korea. J Korean Assoc Oral Maxillofac Surg 2020 
lowed by penicillin with beta-lactamase inhibitors (18.76\%), metronidazole $(12.29 \%)$, second- to fourth-generation cephalosporins (11.52\%), and first-generation cephalosporins (8.61\%). Penicillin accounted for a markedly high proportion of the antibiotics prescribed after incisor extraction and molar extraction, at $46.47 \%$ and $47.21 \%$, respectively. On the other hand, the proportion of penicillin prescribed after extraction of completely impacted teeth was merely $29.55 \%$, while penicillin with beta-lactamase inhibitors accounted for $29.36 \%$ and second- to fourth-generation cephalosporins accounted for $19.13 \%$ of these cases. These outcomes suggest that dentists prescribe broad-spectrum antibiotics more often after invasive tooth extraction procedures.

\section{Antibiotic prescription rates following tooth extraction}

The rate of antibiotic prescription after tooth extraction in Korean adult patients during the 5-year study period (20112015 ) was $81.85 \%$.(Table 3 ) The rate of such prescriptions was significantly higher in male patients $(82.15 \%)$ than in female patients $(81.49 \%)(P<0.001)$. Based on age, the rate in the 19- to 30-year-old group (83.39\%) was significantly higher than in any other age group $(P<0.001)$. Based on household income, the rate of antibiotic prescription was highest in the 'medium-low income' group $(P<0.001)$ and healthy individuals were more frequently prescribed antibiotics than individuals with diabetes and hypertension $(P<0.001)$. The rate of antibiotic prescription was highest in $2015(83.00 \%)$ while the lowest rate was in $2011(80.11 \%)(P<0.001)$. Moreover, while the rate of antibiotic prescription was high-

Table 3. Characteristics according to antibiotic prescription after tooth extraction

\begin{tabular}{|c|c|c|c|c|c|c|}
\hline \multirow{3}{*}{ Variable } & \multirow{3}{*}{ Division } & \multicolumn{4}{|c|}{ Antibiotic prescription } & \multirow{3}{*}{$P$-value } \\
\hline & & \multicolumn{2}{|c|}{ Yes } & \multicolumn{2}{|c|}{ No } & \\
\hline & & $\mathrm{n}$ & $\%$ & $\mathrm{n}$ & $\%$ & \\
\hline All & & 412,308 & 81.85 & 91,417 & 18.15 & \\
\hline \multirow[t]{2}{*}{ Sex } & Male & 227,756 & 82.15 & 49,491 & 17.85 & $<0.001$ \\
\hline & Female & 184,552 & 81.49 & 41,926 & 18.51 & \\
\hline \multirow[t]{6}{*}{ Age (yr) } & $19-30$ & 76,123 & 83.39 & 15,161 & 16.61 & $<0.001$ \\
\hline & $31-40$ & 48,963 & 83.37 & 9,769 & 16.63 & \\
\hline & $41-50$ & 72,278 & 83.10 & 14,700 & 16.90 & \\
\hline & $51-60$ & 93,888 & 82.13 & 20,428 & 17.87 & \\
\hline & $61-70$ & 64,387 & 80.27 & 15,827 & 19.73 & \\
\hline & $\geq 71$ & 56,669 & 78.49 & 15,532 & 21.51 & \\
\hline \multirow[t]{5}{*}{ Household income } & Low & 67,428 & 82.02 & 14,784 & 17.98 & $<0.001$ \\
\hline & Medium-low & 65,325 & 82.29 & 14,062 & 17.71 & \\
\hline & Medium & 77,900 & 82.17 & 16,904 & 17.83 & \\
\hline & Medium-high & 90,833 & 82.08 & 19,825 & 17.92 & \\
\hline & High & 110,822 & 81.09 & 25,842 & 18.91 & \\
\hline \multirow[t]{2}{*}{ Diabetes mellitus } & Yes & 113,826 & 80.43 & 27,697 & 19.57 & $<0.001$ \\
\hline & No & 298,482 & 82.41 & 63,720 & 17.59 & \\
\hline \multirow[t]{2}{*}{ Hypertension } & Yes & 139,046 & 80.39 & 33,922 & 19.61 & $<0.001$ \\
\hline & No & 273,262 & 82.62 & 57,495 & 17.38 & \\
\hline \multirow[t]{2}{*}{ Type of dental institution } & Dental hospital & 39,571 & 81.79 & 8,812 & 18.21 & 0.697 \\
\hline & Dental clinic & 372,737 & 81.86 & 82,605 & 18.14 & \\
\hline \multirow[t]{2}{*}{ Region of dental institution } & Urban & 372,084 & 81.85 & 82,484 & 18.15 & 0.884 \\
\hline & Rural & 40,224 & 81.83 & 8,933 & 18.17 & \\
\hline \multirow[t]{5}{*}{ Year of prescription } & 2011 & 78,295 & 80.11 & 19,445 & 19.89 & $<0.001$ \\
\hline & 2012 & 78,998 & 81.16 & 18,333 & 18.84 & \\
\hline & 2013 & 82,282 & 82.14 & 17,893 & 17.86 & \\
\hline & 2014 & 84,997 & 82.70 & 17,777 & 17.30 & \\
\hline & 2015 & 87,736 & 83.00 & 17,969 & 17.00 & \\
\hline \multirow[t]{6}{*}{ Type of tooth extraction } & Incisor extraction & 48,140 & 77.03 & 14,353 & 22.97 & $<0.001$ \\
\hline & Molar extraction & 268,549 & 81.94 & 59,181 & 18.06 & \\
\hline & Complicated extraction $^{1}$ & 42,808 & 84.00 & 8,152 & 16.00 & \\
\hline & Simply impacted tooth & 14,338 & 84.35 & 2,661 & 15.65 & \\
\hline & Complexly impacted tooth ${ }^{2}$ & 16,331 & 86.03 & 2,651 & 13.97 & \\
\hline & Completely impacted tooth ${ }^{3}$ & 22,142 & 83.36 & 4,419 & 16.64 & \\
\hline
\end{tabular}

${ }^{1}$ Cases where simple extraction was not possible due to root hypertrophy, root dilacerations, or osseointegration.

${ }^{2}$ Cases involving tooth splitting.

${ }^{3}$ Simultaneous tooth splitting and ostectomy of impacted tooth with $\geq 2 / 3$ of crown impacted into alveolar bone.

$P$-value calculated using chi-squared test.

Yoon-Young Choi: Prescription of antibiotics after tooth extraction in adults: a nationwide study in Korea. J Korean Assoc Oral Maxillofac Surg 2020 
est after complexly impacted tooth extraction (86.03\%), the rate was significantly lower after incisor extraction (77.03\%) $(P<0.001)$. There was no significant difference in the rate of antibiotic prescription based on the type or the region of dental institution.

\section{Broad-spectrum antibiotic prescription after tooth extraction}

Among the antibiotics prescribed after tooth extraction, the proportion of broad-spectrum antibiotics was calculated to be $45.88 \%$. (Table 4 ) While there were no significant differences in the proportion of broad-spectrum antibiotic prescription based on sex and household income, the rate of broad-spectrum antibiotic prescription in the 19- to 30-yearold group was highest among all age groups $(P<0.001)$. Fur- thermore, the rate of broad-spectrum antibiotic prescription was significantly higher in patients without systemic disease than in those with diabetes or hypertension $(P<0.001)$, was significantly higher in dental hospitals than in dental clinics $(P<0.001)$, and was significantly higher in rural areas than in urban areas $(P<0.001)$. Lastly, the rate of broad-spectrum antibiotic prescription was significantly higher in 2015 and after completely impacted tooth extraction $(P<0.001)$.

\section{Factors affecting broad-spectrum antibiotic prescription}

In order to identify the factors affecting broad-spectrum antibiotic prescription, multivariate logistic regression analysis was performed. Using a backward elimination method with odds ratios, we aimed to identify the most appropriate model. Diabetes and hypertension were eliminated in each step, leav-

Table 4. Characteristics according to broad-spectrum antibiotic prescription after tooth extraction

\begin{tabular}{|c|c|c|c|c|c|c|}
\hline \multirow{3}{*}{ Variable } & \multirow{3}{*}{ Division } & \multicolumn{4}{|c|}{ Antibiotics } & \multirow{3}{*}{$P$-value } \\
\hline & & \multicolumn{2}{|c|}{ Broad-spectrum } & \multicolumn{2}{|c|}{ Narrow-spectrum } & \\
\hline & & $\mathrm{n}$ & $\%$ & $\mathrm{n}$ & $\%$ & \\
\hline All & & 189,167 & 45.88 & 223,141 & 54.12 & \\
\hline \multirow[t]{2}{*}{ Sex } & Male & 104,609 & 45.93 & 123,147 & 54.07 & 0.472 \\
\hline & Female & 84,558 & 45.82 & 99,994 & 54.18 & \\
\hline \multirow{6}{*}{ Age (yr) } & $19-30$ & 36,299 & 47.68 & 39,824 & 52.32 & $<0.001$ \\
\hline & $31-40$ & 22,601 & 46.16 & 26,362 & 53.84 & \\
\hline & $41-50$ & 32,452 & 44.90 & 39,826 & 55.10 & \\
\hline & $51-60$ & 42,614 & 45.39 & 51,274 & 54.61 & \\
\hline & $61-70$ & 29,511 & 45.83 & 34,876 & 54.17 & \\
\hline & $\geq 71$ & 25,690 & 45.33 & 30,979 & 54.67 & \\
\hline \multirow[t]{5}{*}{ Household income } & Low & 31,157 & 46.21 & 36,271 & 53.79 & 0.180 \\
\hline & Medium-low & 29,962 & 45.87 & 35,363 & 54.13 & \\
\hline & Medium & 35,719 & 45.85 & 42,181 & 54.15 & \\
\hline & Medium-high & 41,409 & 45.59 & 49,424 & 54.41 & \\
\hline & High & 50,920 & 45.95 & 59,902 & 54.05 & \\
\hline \multirow[t]{2}{*}{ Diabetes mellitus } & Yes & 51,524 & 45.27 & 62,302 & 54.73 & $<0.001$ \\
\hline & No & 137,643 & 46.11 & 160,839 & 53.89 & \\
\hline \multirow[t]{2}{*}{ Hypertension } & Yes & 63,209 & 45.46 & 75,837 & 54.54 & $<0.001$ \\
\hline & No & 125,958 & 46.09 & 147,304 & 53.91 & \\
\hline \multirow[t]{2}{*}{ Type of dental institution } & Dental hospital & 23,430 & 59.21 & 16,141 & 40.79 & $<0.001$ \\
\hline & Dental clinic & 165,737 & 44.46 & 207,000 & 55.54 & \\
\hline \multirow[t]{2}{*}{ Region of dental institution } & Urban & 170,240 & 45.75 & 201,844 & 54.25 & $<0.001$ \\
\hline & Rural & 18,927 & 47.05 & 21,297 & 52.95 & \\
\hline \multirow[t]{5}{*}{ Year of prescription } & 2011 & 33,733 & 42.33 & 45,966 & 57.67 & $<0.001$ \\
\hline & 2012 & 33,184 & 41.84 & 46,127 & 58.16 & \\
\hline & 2013 & 36,203 & 43.84 & 46,379 & 56.16 & \\
\hline & 2014 & 41,211 & 48.30 & 44,119 & 51.70 & \\
\hline & 2015 & 45,407 & 51.50 & 42,764 & 48.50 & \\
\hline \multirow[t]{6}{*}{ Type of tooth extraction } & Incisor extraction & 21,195 & 44.03 & 26,945 & 55.97 & $<0.001$ \\
\hline & Molar extraction & 119,012 & 44.32 & 149,537 & 55.68 & \\
\hline & Complicated extraction ${ }^{1}$ & 20,668 & 48.28 & 22,140 & 51.72 & \\
\hline & Simply impacted tooth & 6,920 & 48.26 & 7,418 & 51.74 & \\
\hline & Complexly impacted tooth ${ }^{2}$ & 8,557 & 52.40 & 7,774 & 47.60 & \\
\hline & Completely impacted tooth ${ }^{3}$ & 12,815 & 57.88 & 9,327 & 42.12 & \\
\hline
\end{tabular}

${ }^{1}$ Cases where simple extraction was not possible due to root hypertrophy, root dilacerations, or osseointegration.

${ }^{2}$ Cases involving tooth splitting.

${ }^{3}$ Simultaneous tooth splitting and ostectomy of impacted tooth with $\geq 2 / 3$ of crown impacted into alveolar bone.

$P$-value calculated using t-test and chi-squared test.

Yoon-Young Choi: Prescription of antibiotics after tooth extraction in adults: a nationwide study in Korea. J Korean Assoc Oral Maxillofac Surg 2020 
ing seven explanatory variables including sex, age, household income, type and region of dental institution, year of prescription, and type of tooth extraction that were included in the final analysis.(Table 5)

According to the final regression model (Table 5), females were prescribed broad-spectrum antibiotics at a lower rate than males $(P<0.01)$. All older age groups, compared to the 19- to 30-year-old group, also exhibited a higher likelihood of being prescribed broad-spectrum antibiotics (all $P<0.001$ ). Furthermore, compared with the 'low' household income group, the 'medium-high' and 'high' household income groups exhibited a reduced probability of receiving broadspectrum antibiotic prescriptions $(P<0.01)$, and the probability of broad-spectrum antibiotic prescriptions in dental clinics was lower than in dental hospitals $(P<0.001)$. Based on the region, dental institutions in rural areas exhibited a greater rate of antibiotic prescriptions than those in urban areas $(P<0.001)$. While the probability of broad-spectrum antibiotic prescription was lower in 2012 than in $2011(P<0.001)$, the rates thereof were higher in 2013-2015 ( $P<0.001$ for all). Lastly, compared to incisor extraction, other invasive techniques resulted in a higher chance of broad-spectrum antibiotic prescription $(P<0.01$ for all).

\section{Discussion}

This study utilized a large-scale dataset of 503,725 cases of tooth extraction to evaluate patterns of antibiotic prescription after tooth extraction and factors affecting broad-spectrum antibiotic prescription in Korea. No previous study has performed a nationwide, large-scale cohort analysis of antibiotics prescribed after tooth extraction in Korean dental clinics and hospitals.

The most frequently prescribed antibiotic after tooth extraction was penicillin (45.25\%), which was in agreement with similar studies in South Africa, Germany, and the United States ${ }^{9,12,14}$. Among these studies, the South African study ${ }^{14}$ reported that the rate of penicillin prescription was $80.9 \%$,

Table 5. Logistic regression of broad-spectrum antibiotic prescription after tooth extraction

\begin{tabular}{|c|c|c|c|c|c|c|}
\hline Division & B & SE & $\beta$ & OR & $\mathrm{CI}$ & $P$-value \\
\hline Intercept & 0.034 & 0.020 & & & & 0.083 \\
\hline \multicolumn{7}{|l|}{ Sex $($ male $=1)$} \\
\hline Female & -0.017 & 0.006 & -0.005 & 0.983 & $0.971-0.995$ & 0.007 \\
\hline \multicolumn{7}{|l|}{ Age (yr) (19-30 yr=1) } \\
\hline $31-40$ & 0.065 & 0.012 & 0.012 & 1.067 & $1.042-1.093$ & $<0.001$ \\
\hline $41-50$ & 0.091 & 0.012 & 0.019 & 1.095 & $1.070-1.120$ & $<0.001$ \\
\hline $51-60$ & 0.121 & 0.011 & 0.028 & 1.129 & $1.104-1.154$ & $<0.001$ \\
\hline $61-70$ & 0.141 & 0.012 & 0.028 & 1.152 & $1.125-1.180$ & $<0.001$ \\
\hline$\geq 71$ & 0.110 & 0.013 & 0.021 & 1.116 & $1.088-1.145$ & $<0.001$ \\
\hline \multicolumn{7}{|l|}{ Household income $($ low $=1)$} \\
\hline Medium-low & -0.022 & 0.011 & -0.004 & 0.979 & $0.958-1.000$ & 0.054 \\
\hline Medium & -0.018 & 0.011 & -0.004 & 0.982 & $0.961-1.002$ & 0.084 \\
\hline Medium-high & -0.033 & 0.010 & -0.008 & 0.968 & $0.948-0.987$ & 0.001 \\
\hline High & -0.030 & 0.010 & -0.007 & 0.971 & $0.952-0.990$ & 0.003 \\
\hline \multicolumn{7}{|c|}{ Type of dental institution (dental hospital=1) } \\
\hline Dental clinic & -0.525 & 0.011 & -0.085 & 0.592 & $0.579-0.605$ & $<0.001$ \\
\hline \multicolumn{7}{|c|}{ Region of dental institution (urban $=1$ ) } \\
\hline Rural & 0.100 & 0.011 & 0.016 & 1.105 & $1.082-1.128$ & $<0.001$ \\
\hline \multicolumn{7}{|l|}{ Year of prescription $(2011=1)$} \\
\hline 2012 & -0.036 & 0.010 & -0.008 & 0.964 & $0.945-0.984$ & $<0.001$ \\
\hline 2013 & 0.044 & 0.010 & 0.010 & 1.045 & $1.025-1.066$ & $<0.001$ \\
\hline 2014 & 0.221 & 0.010 & 0.049 & 1.247 & $1.223-1.272$ & $<0.001$ \\
\hline 2015 & 0.351 & 0.010 & 0.079 & 1.420 & $1.393-1.448$ & $<0.001$ \\
\hline \multicolumn{7}{|c|}{ Type of tooth extraction (incisor extraction=1) } \\
\hline Molar extraction & 0.027 & 0.010 & 0.007 & 1.027 & $1.007-1.048$ & 0.009 \\
\hline Complicated extraction $^{1}$ & 0.181 & 0.014 & 0.030 & 1.198 & $1.167-1.231$ & $<0.001$ \\
\hline Simply impacted tooth & 0.207 & 0.021 & 0.021 & 1.230 & $1.181-1.280$ & $<0.001$ \\
\hline Complexly impacted tooth ${ }^{2}$ & 0.315 & 0.020 & 0.034 & 1.370 & $1.318-1.425$ & $<0.001$ \\
\hline Completely impacted tooth ${ }^{3}$ & 0.509 & 0.019 & 0.063 & 1.664 & $1.604-1.725$ & $<0.001$ \\
\hline
\end{tabular}

(B: unstandardized beta, SE: standardized error, $\beta$ : standardized beta, OR: odds ratio, CI: confidence interval)

${ }^{1}$ Cases where simple extraction was not possible due to root hypertrophy, root dilacerations, or osseointegration.

${ }^{2}$ Cases involving tooth splitting.

${ }^{3}$ Simultaneous tooth splitting and ostectomy of impacted tooth with $\geq 2 / 3$ of crown impacted into alveolar bone.

$P$-value calculated by logistic regression analysis.

Variables removed on the model: diabetes mellitus and hypertension.

Yoon-Young Choi: Prescription of antibiotics after tooth extraction in adults: a nationwide study in Korea. J Korean Assoc Oral Maxillofac Surg 2020 
which was markedly higher than in Korea. The values in the German study (51.4\%) and the American study (68\%) were similar to that noted in Korea in the present study ${ }^{9,12}$, but those studies assessed the rate of antibiotic prescription for all dental procedures, not limited to tooth extractions. The second-most frequently prescribed antibiotic varied largely among the different countries. While penicillin with betalactamase inhibitors was the second-most frequently used antibiotic in this study (18.76\%), clindamycin was the secondmost frequently prescribed antibiotic in both the German (34.9\%) and American (15.64\%) studies ${ }^{9,12}$. Moreover, dental clinics in the Czech Republic also frequently prescribed clindamycin $(25.1 \%)^{7}$. The use of lincomycin-derivatives (including clindamycin) in our study was merely $0.21 \%$, and was $<5 \%$ in the UK and Norway, showing varying frequencies among different countries ${ }^{3,23}$. In South Africa, while macrolides were the second-most frequently prescribed antibiotic following tooth extraction, they merely accounted for $3.3 \%$ of all prescribed antibiotics ${ }^{14}$. In the UK, the secondmost frequently prescribed antibiotic was metronidazole, accounting for $28.3 \%$ of all antibiotic prescriptions ${ }^{23}$. While metronidazole was the third-most frequently prescribed antibiotic in our study $(12.29 \%)$, the frequencies of metronidazole prescription in Germany, the US, and South Africa were all $<1 \% \%^{9,12,14}$. Thus, while the use of penicillin as the primary antibiotic in dental settings seems to be the general rule, the use of other antibiotics was markedly variable across countries. These differences are likely due to differences in educational programs, health policies, demographics, and clinical experiences. Furthermore, in this study, the more invasive tooth extraction techniques resulted in a greater frequency of broad-spectrum antibiotic prescription (i.e., penicillins with beta-lactamase inhibitors or second- to fourth-generation cephalosporins), and these outcomes were in agreement with a previous study ${ }^{17}$.

In the present study, the rate of antibiotic prescription after tooth extraction was $81.85 \%$, which was markedly higher than the $11.0 \%$ reported in the South African study ${ }^{14}$. In Korea, the rate of antibiotic prescription even exceeded $80 \%$ after exposed molar extraction, while the highest rate in the South African study was $54.5 \%$ (after impacted tooth extraction in patients with chronic disease). In an Australian study, the rate of antibiotic prescription after third molar extraction ranged between $19 \%$ and $75 \%$ depending on associated symptoms ${ }^{24}$, while approximately $25 \%$ of patients in a Belgian study were given antibiotics after surgical extraction of a third molar $^{20}$. Thus, the rate of antibiotic prescrip- tion in Korea was markedly higher than in other countries, which is consistent with another Korean study that previously described habitual antibiotic prescription after tooth extraction without consideration for potential misuse ${ }^{16}$. Moreover, $45.88 \%$ of antibiotics prescribed after tooth extraction were broad-spectrum antibiotics, raising concerns about the potential misuse of antibiotics.

In this study, the rate of antibiotic prescription was higher in males than in females, as previously reported ${ }^{17}$. However, there was no significant difference in the proportion of broadspectrum antibiotic prescription between males and females. Furthermore, a younger age was associated with higher rates of both antibiotic prescription and broad-spectrum antibiotic prescription. This is likely because older patients tend to have simple tooth extractions due to periodontal disease while tooth extractions in younger patients often involve invasive techniques to extract impacted teeth, such as third molars.

The rates of overall antibiotic and broad-spectrum antibiotic prescription in patients with systemic disease (diabetes and hypertension) were lower than in healthy individuals, although this phenomenon may be partly attributed to a large proportion of patients with systemic disease being elderly who have lower rates of antibiotic prescription as described earlier. Thus, diabetes and hypertension were not significant factors affecting the rate of broad-spectrum antibiotic prescription in a regression model that incorporated all identified confounders, including age. Furthermore, patients with systemic disease sometimes use medications prescribed from their medical clinics rather than dental clinics, which may have contributed to the low rate of antibiotic prescription.

Comparison of antibiotic usage between dental clinics and dental hospitals showed that while there was no difference in the rate of antibiotic prescription, dental hospitals exhibited a greater frequency of broad-spectrum antibiotic prescription. This is likely because cases involving invasive procedures with a high risk of dental infection are often transferred from dental clinics to dental hospitals. Compared to 2011, rates of both antibiotic prescription and broad-spectrum antibiotic prescription were higher in 2015. While this may be due to an increase in antibiotic prescription over time, it may also be explained by the increasing age of participants. Therefore, temporal changes in antibiotic prescription habits cannot be characterized solely based on the findings of our study and additional time series analyses are required.

The use of antibiotics should be limited to severe cases (i.e., those with suspected dental infections), and more specifically, the use of broad-spectrum antibiotics should be mini- 
mized $^{13,21}$. Recently, misuse of antibiotics has been highlighted as the main reason for increased antibiotic-resistance ${ }^{10,25}$. In an effort to investigate factors relating to the prescription of broad-spectrum antibiotics after tooth extraction, the following criteria were concluded to be statistically significant: sex (male), older age, household income (low), type of dental institution (dental hospital), region of dental institution (rural), year of prescription (2015), and type of tooth extraction (completely impacted tooth extraction).

Despite these findings, there were some limitations to this study. First, only antibiotics prescribed on the day of the tooth extraction procedure were included in the analysis. Thus, any antibiotic prescribed prior to or after the date of surgery was not taken into consideration. In addition, tooth extraction for orthodontic treatment is not covered by the NHIS, and those procedures were therefore were not included in this study. Moreover, since the NHIS dataset primarily serves administrative purposes, information that may be crucial to a research study might not always be available. For example, the specific name of tooth extracted or the reason for tooth extraction were not included. Lastly, the large number of participants in this study allows for more accurate representation of the status of antibiotic prescription in Korean dental institutions, but also makes the $P$-values exceedingly small, which may lead to biased conclusions. Despite these limitations, this study utilized the NHIS-NSC dataset-which is representative of the entire Korean population - to analyze antibiotic prescription patterns after tooth extraction while controlling for confounders such as sociodemographic variables and systemic disease. Using this resource minimizes recall bias and allows for accurate representation of the course of prescription and treatment provided to patients, making the outcomes of this study highly reliable.

\section{Conclusion}

This study demonstrates that the rate of antibiotic prescription after tooth extraction in Korea is higher relative to other countries, and broad-spectrum antibiotics are also more frequently used. These trends may be key contributors to antibiotic resistance. Our findings highlight the necessity of a standardized guideline for antibiotic prescription in dental clinics and the need for individual clinicians to minimize unnecessary antibiotic prescription, particularly following tooth extraction. Additional studies should be performed to describe changes in the rates of antibiotic prescription as well as to understand the mechanisms contributing to the high rate of antibiotic prescription in Korea.

\section{Author's Contributions}

Y.Y.C. contributed to the design, data acquisition and analysis, and drafted the manuscript.

\section{Acknowledgements}

This study was performed using NHIS-NSC data (No. NHIS-2019-2-117).

\section{Ethics Approval and Consent to Participate}

This study was performed after receiving ethics approval from the Public Institutional Review Board Committee (P01201901-21-004). Data from the NHIS-NSC are reconstituted data that do not include personal identification, and thus the need for obtaining informed consent from subjects was waived by the institutional review board committee.

\section{Conflict of Interest}

No potential conflict of interest relevant to this article was reported.

\section{References}

1. Dar-Odeh N, Ryalat S, Shayyab M, Abu-Hammad O. Analysis of clinical records of dental patients attending Jordan University Hospital: documentation of drug prescriptions and local anesthetic injections. Ther Clin Risk Manag 2008;4:1111-7.

2. Yingling NM, Byrne BE, Hartwell GR. Antibiotic use by members of the American Association of Endodontists in the year 2000: report of a national survey. J Endod 2002;28:396-404.

3. Al-Haroni M, Skaug N. Incidence of antibiotic prescribing in dental practice in Norway and its contribution to national consumption. J Antimicrob Chemother 2007;59:1161-6.

4. World Health Organization. Antimicrobial resistance: global report on surveillance. Geneva: World Health Organization; 2014.

5. de Kraker ME, Stewardson AJ, Harbarth S. Will 10 million people die a year due to antimicrobial resistance by 2050 ? PLoS Med 2016;13:e1002184.

6. Lee JR, Kim YS, Chang WS, Park OS, Lee YK. Antimicrobial resistance of Staphylococcus aureus isolated from Korean oral cavity. Korean J Oral Maxillofac Pathol 2011;35:31-6.

7. Pipalova R, Vlcek J, Slezak R. The trends in antibiotic use by general dental practitioners in the Czech Republic (2006-2012). Int Dent J 2014;64:138-43.

8. Marra F, George D, Chong M, Sutherland S, Patrick DM. Antibiotic prescribing by dentists has increased: Why? J Am Dent Assoc 2016;147:320-7.

9. Durkin MJ, Hsueh K, Sallah YH, Feng Q, Jafarzadeh SR, Munshi $\mathrm{KD}$, et al. An evaluation of dental antibiotic prescribing practices in the United States. J Am Dent Assoc 2017;148:878-86. 
10. Oberoi SS, Dhingra C, Sharma G, Sardana D. Antibiotics in dental practice: how justified are we. Int Dent J 2015;65:4-10.

11. Cope AL, Francis NA, Wood F, Chestnutt IG. Antibiotic prescribing in UK general dental practice: a cross-sectional study. Community Dent Oral Epidemiol 2016;44:145-53.

12. Halling F, Neff A, Heymann P, Ziebart T. Trends in antibiotic prescribing by dental practitioners in Germany. J Craniomaxillofac Surg 2017;45:1854-9.

13. Holen Ø, Alberg T, Blix HS, Smith I, Neteland MI, Eriksen HM. Broad-spectrum antibiotics in Norwegian hospitals. Tidsskr Nor Laegeforen 2017;137:362-6.

14. Lalloo R, Solanki G, Ramphoma K, Myburgh NG. Antibioticprescribing patterns of South African dental practitioners following tooth extractions. J Investig Clin Dent 2017;8:e12247.

15. Lodi G, Figini L, Sardella A, Carrassi A, Del Fabbro M, Furness S. Antibiotics to prevent complications following tooth extractions. Cochrane Database Syst Rev 2012;11:CD003811.

16. Jo SH, Alrashdan M, Choung HU, Pang KM, Park JC, Kim SM, et al. Literature review of antibiotics prescription in general dental and oral-maxillofacial surgical practice. J Korean Assoc Oral Maxillofac Surg 2009;35:164-9.

17. Kim H, Kim MK, Choi H. Factors affecting the rate of antibiotic prescription in dental practices. J Korean Acad Oral Health 2017;41:28-35

18. Lee JH, Oh JY, Youk TM, Jeong SN, Kim YT, Choi SH. Association between periodontal disease and non-communicable diseases: a 12-year longitudinal health-examinee cohort study in South Korea. Medicine (Baltimore) 2017;96:e7398.

19. World Health Organization. Guidelines for ATC classification and
DDD assignment 2019. Geneva: World Health Organization; 2018. 20. Mainjot A, D'Hoore W, Vanheusden A, Van Nieuwenhuysen JP. Antibiotic prescribing in dental practice in Belgium. Int Endod J 2009;42:1112-7.

21. Blommaert A, Coenen S, Gielen B, Goossens H, Hens N, Beutels P. Patient and prescriber determinants for the choice between amoxicillin and broader-spectrum antibiotics: a nationwide prescriptionlevel analysis. J Antimicrob Chemother 2013;68:2383-92.

22. Shapiro DJ, Hicks LA, Pavia AT, Hersh AL. Antibiotic prescribing for adults in ambulatory care in the USA, 2007-09. J Antimicrob Chemother 2014;69:234-40.

23. Karki AJ, Holyfield G, Thomas D. Dental prescribing in Wales and associated public health issues. Br Dent J 2011;210:E21.

24. Jaunay T, Sambrook P, Goss A. Antibiotic prescribing practices by South Australian general dental practitioners. Aust Dent J 2000;45:179-86; quiz 214.

25. Garg AK, Agrawal N, Tewari RK, Kumar A, Chandra A. Antibiotic prescription pattern among Indian oral healthcare providers: a cross-sectional survey. J Antimicrob Chemother 2014;69:526-8.

How to cite this article: Choi YY. Prescription of antibiotics after tooth extraction in adults: a nationwide study in Korea. J Korean Assoc Oral Maxillofac Surg 2020;46:49-57. https://doi. org/10.5125/jkaoms.2020.46.1.49 